\title{
PREVALÊNCIA DA LEISHMANIOSE TEGUMENTAR AMERICANA NO NORTE DO ESTADO DO PARANÁ: ASPECTOS CLÍ́NICOS E EPIDEMIOLÓGICOS
}

\author{
Therezinha Monteiro Pullina \\ Edna Maria Vissoci Reicheb
}

\begin{abstract}
RESUMO
Com bases nos registros da Superintendência de Companhias do Ministério da Saúde-Regional do Paraná (SUCAM-PR) de 507 casos de Leishmaniose Tegumentar Americana (LTA) referentes ao periodo de janeiro a dezembro de 1986 e nos registros de 31 pacientes atendidos no Setor de Imunologia Clínica do Hospital Regional do Norte do Paraná-HURNP, no periodo de maio de 1985 a dezembro de 1986, realizou-se um estudo retrospectivo com o objetivo de determinar a prevaléncia e estudar aepidemiologia desta parasitose nos municipios do norte do Estado do Paraná. Os dados revelaram que os municipios de maior porcentagem de casos notificados foram Jussara (18,11\%), Cianorte (12,22\%), Terra Boa (10,65\%), Engenheiro Beltrüo $(5,37 \%)$, Londrina $(5,12 \%)$ e São Jorge do Ivai $(4,33 \%)$. A maioria destes municipios são continuos entre si, formando areas endêmicas principalmente no Vale do Rio Ivai. Analisou-se tambèm 31 casos de L.T.A., relacionando a parasitose com a idade, sexo, cor, local e aspecto clínico das lesöes e profissāo dos pacientes acometidos.
\end{abstract}

PALAVRAS CHAVES: Leishmaniose tegumentar americana; Epidemiologia.

\section{1-INTRODUC̄̄o}

A escassez de informaçбos sobre a prevalência e epidemiologia da L.T.A., levou-nos a realizar um estudo retrospectivo, tendo como objetivo geral determinar a prevalência e estudar a cpidemiologia desta patologia na população de diversos municípios no Norte do Paraná.

Apesar do agente etiológico da Leishmaniose Tegumentar Americana (LTA) só ter sido identificado por volta do início do século $\mathrm{XX}$, muitos pesquisadores acreditam que esta enfermidade seja endèmica há muitos anos no continente americano, talvez mesmo até antes do seu descobrimento

Nos primórdios deste século, com o desbravamento das matas da Região Noroeste do Estado de Sắo Paulo, começaram a afluir inúmeros doentes ao Hospital da Santa Casa da cidade de São Paulo portando lesóes de L.T.A.

Em 1911, SPLENDORE, conseguiu isolar leishmänias das lesōes mucosas desta moléstia e a partir desta época, foi entấo constatada em todos os estados do Brasil (9:739) Com o desbravamento das matas, construçócs de estradas, aberturas de fazendas em regióes agrestes, o homem começou a pagar o seu tributo à doença.

\section{2-MATERIAL E MÉTODOS}

Para este estudo retrospectivo foram levantados os dados notificados mensalmente à Superintendência de Campanhas do Ministério da Saúde (SUCAM), totalizando 507 casos de
L.T.A. diagnosticados em 63 municipios no Norte do Estado do Paraná, no período de janeiro a dezembro de 1986. Nestes registros não há indicação dos métodos diagnósticos nem da forma clínica da doença.

Foram utilizados também, dados referentes a 31 pacientes atendidos no Setor de Imunologia Clínica do Hospital Universitário Regional do Norte do Paraná (HURNPR) durante o período de maio de 1985 a dezembro de 1986.

No setor de Imunologia Clínica do HURNPR, o diagnóstico baseou-se no aspecto clinico đa lesāo e na intradermorreação de Montenegro, que utilizando-se do antígeno de Montenegro, fornecido pelo Instituto Adolfo Lutz, foi considerada positiva com enduração acima de $5 \mathrm{~mm}$ de diāmetro,

\section{3-APRESENTAÇĀO DOS RESULTADOS}

O estudo retrospectivo proposto no presente trabalho, resultou nos dados que serão apresentados nas tabelas 1 a 7

Observa-se que os municípios com maior número de casos comunicados foram Jussara com 92 casos $(18,22 \%)$, Cianorte com 62 casos $(12,22 \%)$, Terra Boa com 54 casos $(10,65 \%)$, Engenheiro Beltrāo com 27 casos (5,35\%), Londrina com 26 casos $(5,12 \%)$, e São Jorge do Ivaí com 22 casos $(4,33 \%)$. Seguem com menor ocorréncia Barbosa Ferraz e Dr. Camargo com 19 casos cada, $(3,74 \%)$, Colorado com 15 casos $(2,95 \%)$, Jaguapitā com 14 casos $(2,76 \%)$ e Lobato

Recebido cm 9/9/87

a Farmacêutica Bioquímica, Especialista em Saúde Pública.

b Departamento de Patologia Aplicada, Legislação e Deontologia - CCS/UEL 
$\operatorname{com} 12 \operatorname{casos}(2,36 \%)$.

Dentre os casos registrados pela SUCAM-PR, $235(46,36 \%)$ ocorreram nos municípios de Jussara, Cianorte, Terra Boa e Engenheiro Beltrão, cidades contínuas entre si, como demonstra a FIG. 1

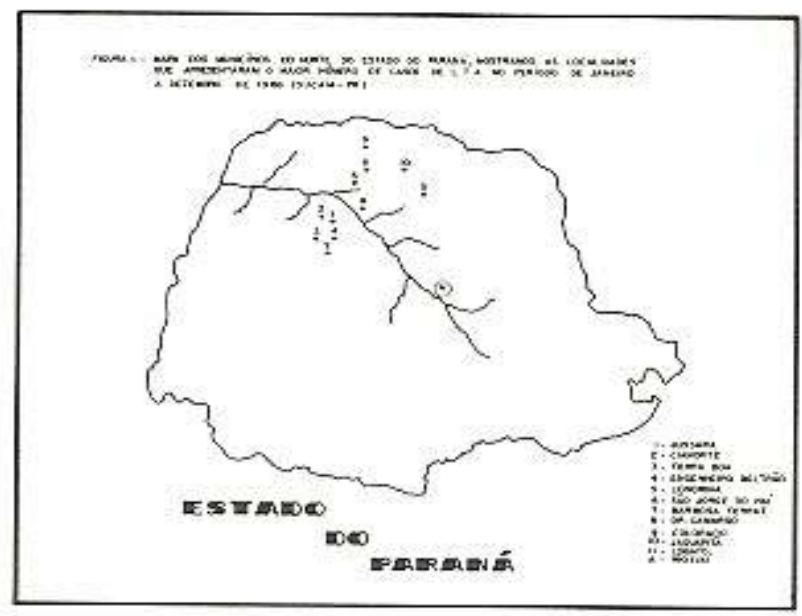

TABELA 2 - Distribuição etária de 31 pacientes com L.T. A. atendidos no Setor de Imunologia Clínica do HURNPR no período de maio de 1985 a dezembro de 1986.

\begin{tabular}{lcr}
\hline IDADE (ANOS) & NO & \\
\hline $0-10$ & 1 & 3,22 \\
$10-20$ & 1 & 3,22 \\
$20-30$ & 7 & 22,58 \\
$30-40$ & 3 & 9,67 \\
$40-50$ & 6 & 19,35 \\
$50-60$ & 6 & 19,35 \\
$60-70$ & 6 & 19,35 \\
70 ou mais & 1 & 3,22 \\
\hline TOTAL & 31 & $100 \%$ \\
\hline
\end{tabular}

A idade dos pacientes atendidos no Setor de Imunologia Clínica do HURNPR variou entre 4 e 75 anos sendo a faixa etária de 20 - 30 anos a que apresentou a maior porcentagem de pacientes acometidos, $22,28 \%$ ).

TABELA 3 - Distribuiçāo quanto ao sexo de 31 pacientes com L.T.A. atendidos no Setor de Imunologia Clínica do HURNPR no periodo de maio de 1985 a 1986.

\begin{tabular}{lcc}
\hline SEXO & NO & $\%$ \\
\cline { 2 - 3 } Masculino & 21 & 67,74 \\
Feminino & 10 & 32,26 \\
\hline TOTAL & 31 & $100 \%$ \\
\hline
\end{tabular}

TABELA 4 - Distribuição quanto a cor dos 31 pacientes com L.T.A. atendidos pelo Setor de Imuno. logia Clínica do HURNPR no período de maio de 1985 a dezembro de 1986.

\begin{tabular}{lrr}
\hline COR & No & $\%$ \\
\hline Negra & 1 & 3,22 \\
Parda & 14 & 45,16 \\
Branca & 16 & 51,61 \\
\hline TOTAL & 31 & $100 \%$ \\
\hline
\end{tabular}

TABELA 5 - Distribuição quanto localização das lesões dos 31 pacientes com L.T.A. atendidos no Setor de Imunologia Clínica do HURNPR no periodo de maio de 1985 a dezembro de 1986

\begin{tabular}{lr}
\hline LOCAL & NO \\
\hline Região Nasal (septo nasal, narinas) & 12 \\
Face & 5 \\
Olhos & 3 \\
Pescoço & 1 \\
Orelhas & 2 \\
MMSS & 8 \\
MMII & 13 \\
Couro cabeludo & 1 \\
Região lombar & 1 \\
\hline
\end{tabular}

A Tabela 5 apresenta os dados referentes a localização das lesôes dos casos analisados, sendo que um mesmo doente podia apresentar mais de uma lesão, atingindo principalmente áreas expostas. Como podemos notar, houve predominância dos membros inferiores (MMII, 13 casos), seguidos da região nasal (12 casos) e membros superiores ( 8 casos).

TABELA 6 - Distribuição quanto ao aspecto clínico das lesōes apresentadas pelos 31 pacientes com L.T.A. atendidos pelo Setor de Imunologia Clínica do HURNPR no período de 1985 a dezembro de 1986.

\begin{tabular}{lr}
\hline FORMAS CXINICAS & No \\
\hline Ulcerada & 16 \\
Pápulo-ulcerada & 1 \\
Crostosa & 10 \\
Vegetante & 1 \\
Verrugosa & 0 \\
Nodulares & 1 \\
Presença de nódulos cervicais & 1 \\
Presença de nódulos inguinais & 4 \\
Eritêmato-papulosa & 3 \\
Vesídulo-papulosa & 1 \\
\hline
\end{tabular}


De acordo com os dados referentes ao aspecto clínico das lesōes apresentadas na Tabela 6 , podemos notar uma predominância na forma ulcerada e crostosa, sendo que um mesmo paciente podia apresentar várias formas clínicas da patologia.

TABELA 7 - Distribuição quanto a profissāo dos 31 pacientes com L.T.A. atendidos pelo Setor de Imunologia Clínica do HURNPR no período de maio de 1985 a dezembro de 1986.

\begin{tabular}{lrr}
\hline PROFISSÃO & N⿳亠 & $\%$ \\
\hline Lavrador & 11 & 35,48 \\
Doméstica & 9 & 29,03 \\
Motorista & 1 & 3,22 \\
Aposentado & 3 & 9,67 \\
Vendedor & 1 & 3,22 \\
Carpinteiro & 1 & 3,22 \\
Pedreiro & 1 & 3,22 \\
Serralheiro & 1 & 3,22 \\
Desconhecida & 2 & 6,45 \\
\hline TOTAL & 31 & $100 \%$ \\
\hline
\end{tabular}

De acordo com a Tabela 7 , pode-se observar uma maior porcentagem de lavradores $(35,48 \%)$, seguida das domésticas $(29,03 \%)$ e aposentados $\operatorname{com}(9,67 \%)$.

\section{4-DISCUSSÃO}

A anátise dos registros feitos pela SUCAM-PR de janciro a dezembro de 1986 , demonstrou que dos 507 pacientes acometidos pela L.T.A., $235(46,36 \%)$ casos, eram provenientes de cidades próximas umas das outras, pertencentes ao Vale do Kio Ivai, formando uma área endêmica. (Tabela 1 e Figura 1).

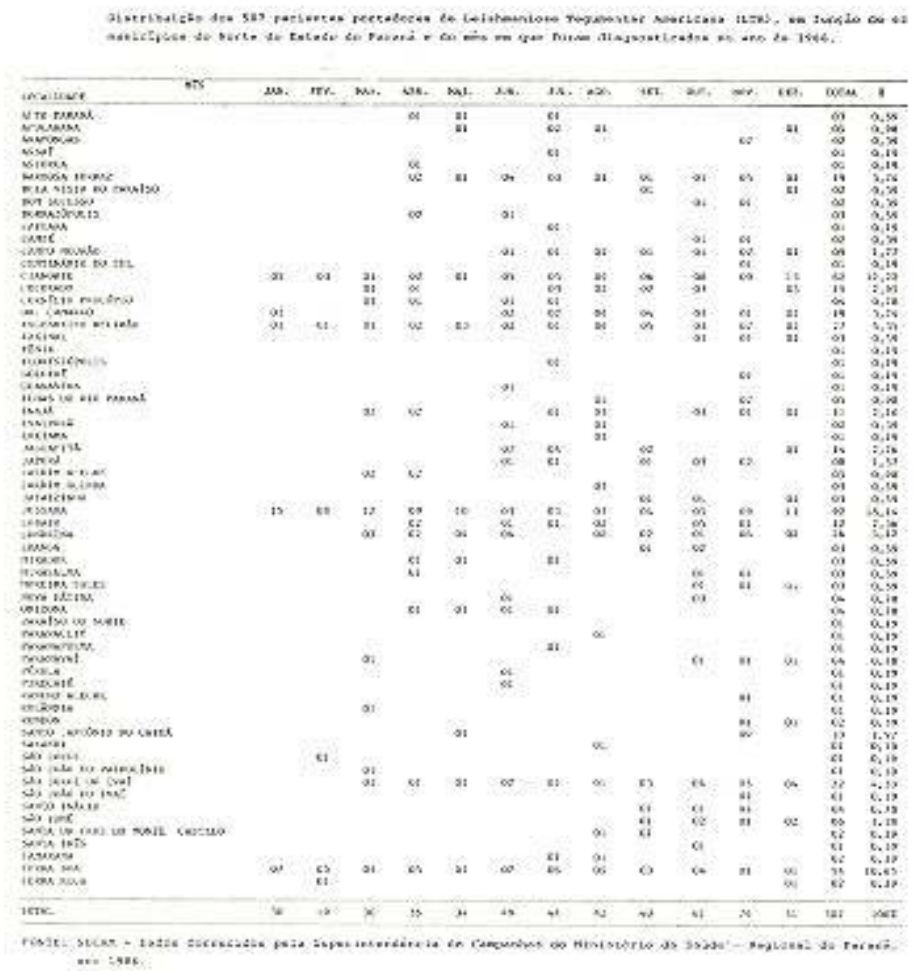

O fato destes municípios serem descontínuos de outras áreas endêmicas, indica que surgiram internamente, condiçōes favoráveis à transmissão que provavelmente seriam o crescente aparecimento de novas fazendas na regiāo, que devem ser analisados como justificativa dos resultados obtidos.

Segundo NEVES (7:764), a L.T.A. atinge o homem em qualquer idade, desde a infância até a senilidade, fato este encontrado neste estudo retrospectivo, onde verificou-se a ocorrência de 01 caso $(3,22 \%)$ em criança abaixo de 10 anos, como também 01 caso $(3,22 \%)$ em paciente com mais de 70 anos. (TABELA 2).

Com relação ao sexo, embora vários autores coloquem a não predileção da L.T.A. por esta variável, encontrou-se uma predominânica do sexo masculino nos 31 casos analisapor serem mais acessiveis à picada do flebótomo transmispossibilidade de exposição profissional (1:330) TABELA 3.

Os resultados apresentados na Tabela 5 e 6 mostram que a maior freqüência das lesōes ocorrem em áreas expostas, por serem mais acessiveis à picada do flebótomo transmissor da doença.

Quando a profissão dos 31 pacientes analisados observou-se um predomínio de lavradores, 11 casos $(35,48 \%)$ seguidos de 9 domésticas $(29,03 \%)$. Este fato provavelmente é explicado por ser comum em nossa regiāo a mulher auxiliar o marido nos trabalhos do campo. Este resultado também foi observado por PIRAINO et alii, (8:107 - 108), quando estudou um surto epidêmico de L.T.A. no município de Lobato, Paraná.

\section{5-CONCLUSÃO}

Levando-se em consideração as limitaçơes do presente estudo retrospectivo, com relaçăo aos dados fornecidos pela 
SUCAM-PR., como também pelo Setor de Imunologia Clinica do HURNPR, o trabalho possibilita chegar às seguintes conclusōes:

- a L.T.A. ocorre com uma prevalência significativa nos municípios do Norte do Paraná, ( 507 casos em 63 municípios no ano de 1986) concentrados principalmente, nos municípios pertencentes ao Vale do Rio Ivaí.
- a L.T.A., embora não tenha predileção por sexo, idade e cor, encontrou-se uma maior porcentagem de pacientes do sexo masculino, adultos e cor branca.

- os pacientes apresentaram um polimorfismo clínico e lesōes que predominam em áreas expostas do corpo.

- os trabalhadores rurais sāo os pacientes mais acometidos pela L.T.A., caracterizando uma doença profissional.

\begin{abstract}
Based both on files from the "Superintendencia de Campanhas do Ministério da Satide Regional do Paraní (SUCAM-PR)" containing 507 cases of Leishmaniose Tegumentar Americana (L.T.A.) referring to the period from January to December 1986, and on the fites from 31 patients treated at the "Setor de Imunologia Clinica do Hospital Regional do Norte do Parana' - HURNP", for the period from May to December 1986, a retrospective study was done in order to determine the prevalence and to study the epidemiology of this parasite in the Municipal districts of North of Parand. The data revealed the municipal districts with greatest of notified cases were Jussara (184\%), (ïanorte (12,22\%), Terra Boa (10,65\%), Engenheiro Beltrāo (5,37\%), Londrina $(5,12 \%)$ and Sāo Jorge do Ivai $(4.33 \%)$. The majority of those districts are neighbours, forming endemic areas mainly in the Ivai River Valley. In addition, 31 cases were analyzed, relating parasitism to age, sex, color, place of lesions, clinical forms and occupation of affected patients.
\end{abstract}

KEY WORDS: Leishmaniose tegumentar americana, Epidemiology.

\title{
REFERẼNCIAS BIBLIOGRÁFICAS
}

1. Amato, NETO, V. Doenças transmissiveis. 2 ed. Rio de Janciro, Guanabara Koogan, 1978. p. 329-334.

2. BEENSON \& MCDERMOTT, ed. Tratado de medicina. $13 \mathrm{ed}$. Rio de Janeiro, Guanabara Koogan, 1973. Tomo I, p. 718-725.

3. BENENSON, A.S. Controle das doenças transmissíveis no homem. 13 ed. OPA da Saúde, 1983. (Relatório Oficial da Associação Americana de Saúde Pública).

4. CHAVES, C. de S. Reacōes de Aglutinação Direta para o soro diagnostico das Leishmanioses. Instituto de Microbiologia da Universidade Federal do Rio de Janciro, 1981. $123 \mathrm{p}$. Tese (Mestrado).

5. GOMES, A. de C. et alii, Aspectos ecológicos da Leishmaniose Tegumentar Americana. 3, Observaçōes naturais sobre o ritmo diário do Psychodopygus intermedius em ambiente florestal e extra florestal. Rev. Saúde Pública, Sāo Paulo, 17:23-30, 1983.
6. GRIMALDI JR., G. Leishmanioses Tegumentares: aspectos clínicos e imunológicos. Mem. Instíluto Osvaldo Cruz, Rio de Janeiro, 77(2): 195-215, abr./jun., 1982.

7. NEVES, J. Diagnostico e tratamento das doenças infectuosas $e$ parasitúrics. 2. ed. Rio de Janeiro, Guanabara Koogan, 1978. p. $761-769$.

8. PIRAINO, R. et alii. Surto epidemiológico de Leishmaniose Tegumentar Americana no município de Lobato-Paraná, Semina, 6(2): 107-109, 1980 .

9. VERONESI, R. Doenças infecciosas e parasitarias, 7 ed, Rio de Janeiro, Guanahara Koogan, 1982. p. 739-752.

10. YOSHIDA, E.L. de A. Leishmaniose tegumentar americana (Reservatórios e Aspectos Toxicologicos do Género Leishmânia. Instituto de Ciēncias Biológicas da Universidade de São Paulo, 1981. 188 p. Dissertaçāo (Mestrado). 